\title{
Long-term instability of fasting plasma glucose predicts mortality in elderly NIDDM patients: the Verona Diabetes Study
}

\author{
M. Muggeo ${ }^{1}$, G. Verlato ${ }^{2}$, E. Bonora ${ }^{1}$, F. Ciani ${ }^{1}$, P. Moghetti ${ }^{1}$, R. Eastman ${ }^{3}$, G. Crepaldi ${ }^{4}$, R. de Marco $^{2}$ \\ ${ }^{1}$ Division of Metabolic Diseases, University of Verona, Verona, Italy \\ ${ }^{2}$ Institute of Medical Statistics, University of Verona, Italy \\ ${ }^{3}$ Division of Diabetes, Endocrinology and Metabolic Diseases, National Institute of Diabetes, Digestive and Kidney Diseases, \\ Bethesda, Maryland, USA \\ ${ }^{4}$ Institute of Internal Medicine, University of Padova, Padova, Italy
}

Summary The aim of this study was to evaluate whether long-term glucose control, as assessed by fasting plasma glucose determinations during 3 years, is a predictor of all-cause mortality in elderly NIDDM patients. Five hundred and sixty-six NIDDM patients attending the Verona Diabetes Clinic, aged 75 years and over, were followed-up from 1 January 1987 to 31 December 1991 to assess all-cause mortality. From their clinical records all fasting plasma glucose determinations available for the years 1984 to 1986 were collected and analysed. Patients were grouped in tertiles according to mean (M-FPG), coefficient of variation (CV-FPG) and trend over time (slope, S-FPG) of fasting plasma glucose during the period of retrospective evaluation. Mortality was assessed by observed/expected ratios, univariate Kaplan-Meier survival analysis and multivariate Poisson regression model. By 31 December 1991, 61 men and 127 women had died. Increased observed/expected ratios were found in women from the top M-FPG tertile, in patients (men and women) from the top CV-FPG tertile and in patients with a S-FPG less than $-0.30 \mathrm{mmol} / 1$ per year (lowest tertile). Patients in the lowest tertile of CV-FPG and in the middle tertile of S-FPG had a reduced mortality risk. Kaplan-Meier survival analysis indicated that patients with high CV-FPG as well as those in tertiles I and III of S-FPG (i.e., those with a definitely negative or definitely positive slope) had an increased probability of dying, without any significant differences between the three tertiles of M-FPG. Poisson regression model showed that CV-FPG, but not MFPG or S-FPG, was an independent significant predictor of mortality. These results suggest that glucose stability needs to be considered along with the absolute level of metabolic control when treating elderly NIDDM patients. [Diabetologia (1995) 38: 672-679]

Key words Non-insulin-dependent diabetes mellitus, mortality, elderly, hyperglycaemia, glucose control.
As the prevalence of diabetes mellitus markedly increases with advancing age [1-5] and elderly people are becoming a growing component of the population in western countries, a considerable proportion of elderly subjects are diabetic and their number is

Received: 10 August 1994 and in revised form: 24 November 1994

Corresponding author: Professor M. Muggeo, Malattie del Metabolismo, Ospedale Civile Maggiore, Piazzale Stefani 1, I-37126 Verona, Italy

Abbreviations: SHU, Social Health Unit; M-FPG, mean fasting plasma glucose; CV-FPG, coefficient of variation of fasting plasma glucose; S-FPG, slope of fasting plasma glucose over time. progressively increasing. In agreement with other studies, we recently observed that in Verona, Italy, the highest prevalence of diabetes is reached between 65 and 79 years in men, and between 75 and 80 years in women [6].

In elderly people diabetes is associated with the highest rates of morbidity and mortality for chronic diabetic complications [7] and for non-diabetes-related diseases [8]. In Verona, we recently found that in diabetic subjects an excess mortality persists in advanced age [6]. This result confirms previous reports $[9,10]$. The true determinants of the excess mortality in elderly subjects with diabetes are poorly understood. In particular, at present it is unknown whether the degree of metabolic control is a major determi- 
Table 1. Main demographic and baseline clinical characteristics of the elderly diabetic cohort

\begin{tabular}{lccc} 
& \multicolumn{2}{c}{ Diabetic patients attending the clinic } & \multicolumn{2}{c}{$\begin{array}{c}\text { Diabetic patients not } \\
\text { attending the clinic }\end{array}$} & Not in the study & 905 \\
\cline { 2 - 3 } Subjects & In the study & 321 & $263(29.1 \%)$ \\
Sex (male) & 566 & $113(35.2 \%)$ & $80.9 \pm 4.4$ \\
Age (years) & $157(27.2 \%)$ & $78.8 \pm 4.4$ & \\
Age (distribution) & $79.8 \pm 2.6$ & $228(71.0 \%)$ & $446(49.3 \%)$ \\
$75-79$ years & $344(60.8 \%)$ & $53(16.5 \%)$ & $290(32.0 \%)$ \\
$80-84$ years & $197(34.8 \%)$ & $40(12.5 \%)$ & $169(18.7 \%)$ \\
$\geq 85$ years & $25(4.4 \%)$ & $12.0 \pm 8.2$ & $10.9 \pm 8.7$ \\
Diabetes duration (years) & $13.2 \pm 7.0$ & $29(9.0 \%)$ & $65(7.4 \%)$ \\
Therapy & & $263(81.9 \%)$ & $782(88.8 \%)$ \\
Diet & $52(9.2 \%)$ & $21(6.5 \%)$ & $19(2.2 \%)$ \\
OHA & $477(84.3 \%)$ & $8(2.5 \%)$ & $15(1.7 \%)$ \\
Insulin + OHA & $27(4.8 \%)$ & $10(1.8 \%)$ & \\
Insulin & & & \\
\hline
\end{tabular}

${ }^{\mathrm{a}}$ Information on therapeutic regimen was missing for 24 subjects not attending the clinic

nant of mortality in elderly diabetic subjects. Such information is crucially related to the question of how intensively to treat elderly diabetic subjects. This is a major public health problem to be addressed, as diabetes of elderly people is an increasing burden on social and medical resources [11].

In the present study we examined whether longterm glucose control, as assessed by retrospective analysis of fasting glucose levels in the 3 years preceding a 5-year mortality follow-up, is a significant determinant of all-cause mortality in diabetic patients aged 75 years and over.

\section{Subjects and methods}

Subjects. The present study is a part of the Verona Diabetes Study [6], a population-based survey which allowed us to identify about 7500 known diabetic subjects who met the diagnostic criteria of the National Diabetes Data Group [12]. These subjects were alive and resident in the area corresponding to the Social Health Unit (SHU) of Verona on 31 December 1986 (baseline). For the purpose of this study, we included all the subjects ( $n=566,157$ men, 409 women) aged 75 years and over, attending the Verona Diabetes Clinic in the years 1984 to 1986 , and of whom at least two fasting plasma glucose determinations for each of these years were available. These subjects were followed-up for the subsequent 5 years (1 January 1987-31 December 1991) to assess all-cause mortality.

The demographic and main baseline clinical characteristics of the cohort under study are summarized in Table 1, together with those of elderly subjects attending the clinic but excluded from the analysis because of a lower number of glucose determinations, as well as those of diabetic patients of the same age not attending the clinic but identified by the Verona Diabetes Study. No substantial differences were found between subjects attending the clinic included and excluded from the analysis, except for marginal differences in age and sex distribution, while slightly larger differences were found with respect
Values are given as mean \pm SD for continuous variables and as absolute frequency, with percent frequency in parentheses, for categorical variables. OHA, Oral hypoglycaemic agents to diabetic subjects not attending the clinic. In the cohort un der study mean age was about 80 years, duration of disease was about 13 year, women were more represented than men, and about $90 \%$ of subjects were treated with oral hypoglycaemic agents, alone or in combination with insulin. All the pa tients of the cohort met the criteria for diagnosis of non-insu lin-dependent (NIDDM) diabetes [12]. In particular, in those treated with insulin, the diagnosis of diabetes had been made after the age of 50 years (mean 61.8 years; range $52-73$ years), and insulin treatment had been initiated at least 10 years after diagnosis of diabetes was made (mean 16.7 years; range $10-$ 25 years).

Fasting glucose determinations. In the patients under study fasting plasma glucose had been repeatedly measured in a consistent fashion during the years preceding the baseline, and the values were available in the clinical records. The latter were carefully reviewed to collect all the plasma glucose levels measured at fasting during the years 1984 to 1986 .

For fasting plasma glucose measurements patients had been instructed to fast after 22.00 hours the previous night. Venous blood had been drawn from an antecubital vein between 07.30 and 08.30 hours, collected in tubes containing EDTA and fluoride and centrifuged within $2 \mathrm{~h}$. Plasma glucose had been assayed with a glucose-oxidase method (Boheringer Mannheim, Mannheim, Germany). Patients who arrived at the Diabetes Clinic later than 08.30 hours or who had eaten in the morning of the day of the visit were instructed to return on another day.

Mortality assessment. The life status of the diabetic cohort identified on 31 December 1986 was ascertained on 31 December 1991 as follows. All the death certificates of the Verona SHU from 1 January 1987 up to 31 December 1991 (about 18,000 ), as well as the mortality records of the Verona SHU Department of Statistics concerning the same time period were carefully reviewed and deaths of diabetic subjects identified on 31 December 1986 by the Verona Diabetes Study [6] were recognized. Deceased diabetic subjects were identified by matching National Social Health Code, name, date and place of birth of subjects in the death certificates and in the mortality records with those of subjects in the diabetes register established with the Verona Diabetes Study on 31 Decem- 
Table 2. Main demographic and baseline clinical characteristics of survivors and deceased in the elderly diabetic cohort

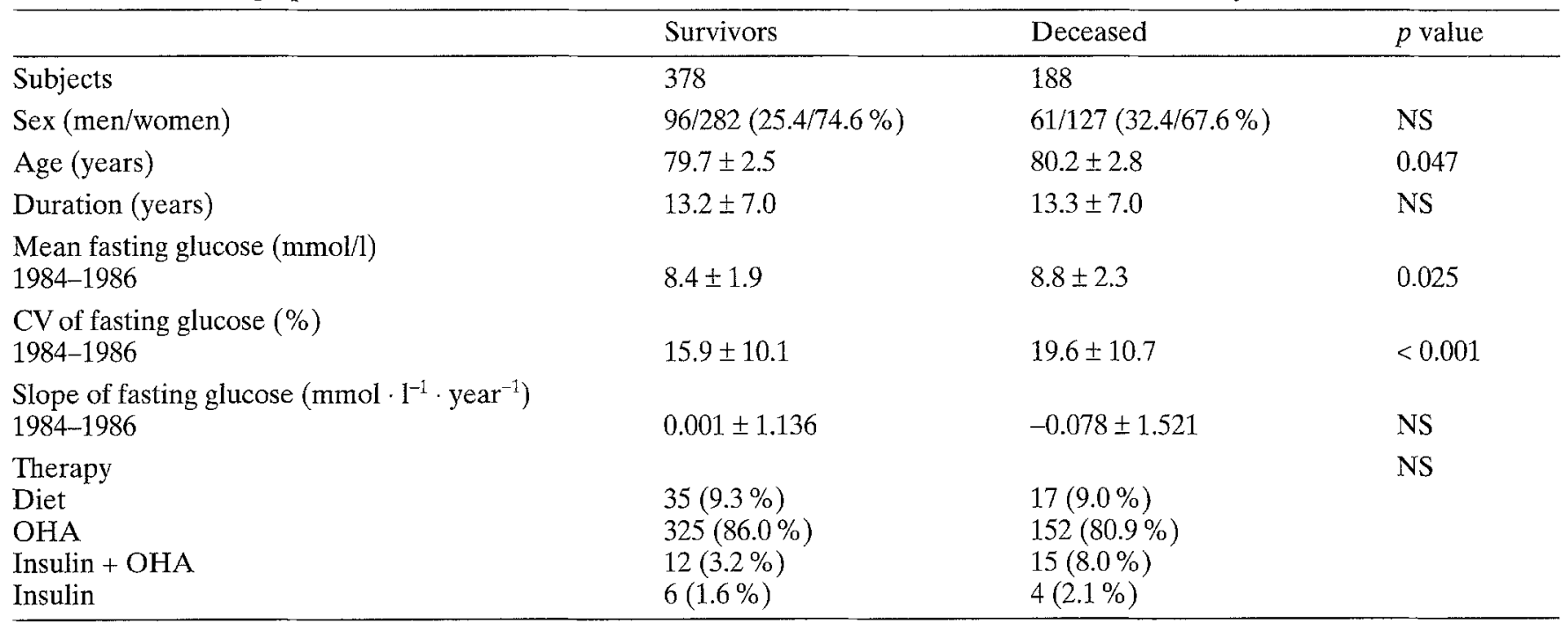

NS, Not significant; OHA, oral hypoglycaemic agents

Values are given as mean \pm SD for continuous variables and as

absolute frequency, with percent frequency in parentheses, for categorical variables

Table 3. Mean fasting plasma glucose (M-FPG), coefficient of variation (CV-FPG) and regression coefficient of fasting plasma glucose over time (S-FPG) as a function of therapeutic regimen in the elderly diabetic cohort during 3 years preceding the mortality follow-up

\begin{tabular}{lccccc}
\hline & $\begin{array}{l}\text { Diet } \\
(n=52)\end{array}$ & $\begin{array}{l}\text { OHA } \\
(n=477)\end{array}$ & $\begin{array}{l}\text { OHA + insulin } \\
(n=27)\end{array}$ & $\begin{array}{l}\text { insulin } \\
(n=10)\end{array}$ \\
\hline M-FPG $(\mathrm{mmol} / \mathrm{l})$ & $6.9 \pm 1.1$ & $8.5 \pm 1.9$ & $11.6 \pm 2.3$ & $11.0 \pm 3.2$ & $<0.001$ \\
CV-FPG $(\%)$ & $13.2 \pm 11.5$ & $16.9 \pm 10.0$ & $24.8 \pm 10.4$ & $26.3 \pm 10.0$ \\
S-FPG $\left(\mathrm{mmol} \cdot \mathrm{l}^{-1} \cdot\right.$ year $\left.^{-1}\right)$ & $-0.03 \pm 1.15$ & $-0.02 \pm 1.24$ & $-0.24 \pm 1.94$ & $0.27 \pm 1.60$ & $\mathrm{NS}$ \\
\hline
\end{tabular}

NS, Not significant; OHA, oral hypoglycaemic agents

Data are reported as mean $\pm \mathrm{SD}$

ber 1986. A list of all the people who moved from Verona during the years 1987-1991 was obtained by the Department of Statistics, the list was checked to identify subjects included in the diabetic cohort followed-up for mortality, their new addresses were obtained, and their life status was ascertained by telephone. Of the initial cohort 21 (3.3\%) subjects who had moved from Verona were not traced, and were arbitrarily considered alive at the end of the follow-up.

Observed deaths were compared with expected deaths, using as reference the general population of the SHU of Verona, after excluding diabetic/subjects. Sex- and 5-year age-specific mortality rates for all causes in non-diabetic subjects were calculated using data obtained from the Verona SHU Department of Statistics.

\section{Statistical analysis}

The total number of glucose determinations available in the clinical records and collected for the analysis was 5048, with an average of 3.0 plasma glucose determinations per subject per year (median 2.7, range 2-6). For each patient the mean (M-FPG) and the coefficient of variation (CV-FPG) of fasting plasma glucose in the 3 years preceding the mortality followup were calculated. In addition, in each individual patient the overall trend of fasting plasma glucose across the 3 years of observation was evaluated through the regression coefficient (slope) of fasting plasma glucose over time (S-FPG). Patients were grouped in tertiles according to M-FPG $(<7.46,7.46-$ 9.16 and $\geq 9.17 \mathrm{mmol} / \mathrm{l}), \quad$ CV-FPG $\quad(<11.2, \quad 11.2-18.4$, $\geq 18.5 \%$ ), and S-FPG (less than $-0.30,-0.30$ to +0.33 , more than $+0.34 \mathrm{mmol} / 1$ per year).

Sex- and age-adjusted mortality risk was evaluated by calculating the observed/expected ratios by means of the computer program PYRS (person-years) version 1.3 [13]. Univariate survival analysis was carried out by Kaplan-Meier method and log-rank test [14]. Multivariate survival analysis was accomplished by Poisson regression model [15]. In the multivariate analysis M-FPG, CV-FPG and S-FPG along with gender, age, diabetes duration and insulin treatment were considered. M-FPG, CV-FPG and S-FPG were always treated as categorical variables in all statistical analyses. In the Poisson regression model insulin treatment was considered as a twolevel categorical variable $(0=$ absent, $1=$ present $)$. Poisson regression model was chosen because the hazard associated with different tertiles of CV-FPG showed a significant interaction with time. The relative risk for continuous variables was calculated on the basis of an increase in the values of $1 \mathrm{SD}$.

Other statistics were carried out by unpaired Student's $t$ test, chi-square analysis, one-way analysis of variance and Pearson's simple correlations [16]. Since the distribution of values of S-FPG was significantly different from normal, Mann-Whitney and Kruskal-Wallis tests were used to analyse this variable [16]. 
Table 4. All-cause mortality in the elderly diabetic cohort during a 5-year follow-up (1 January 1987-31 December 1991), as a function of mean fasting plasma glucose (M-FPG) and its

variation coefficient (CV-FPG) as well as regression coefficient of fasting plasma glucose over time (S-FPG) during 3 years preceding the mortality follow-up

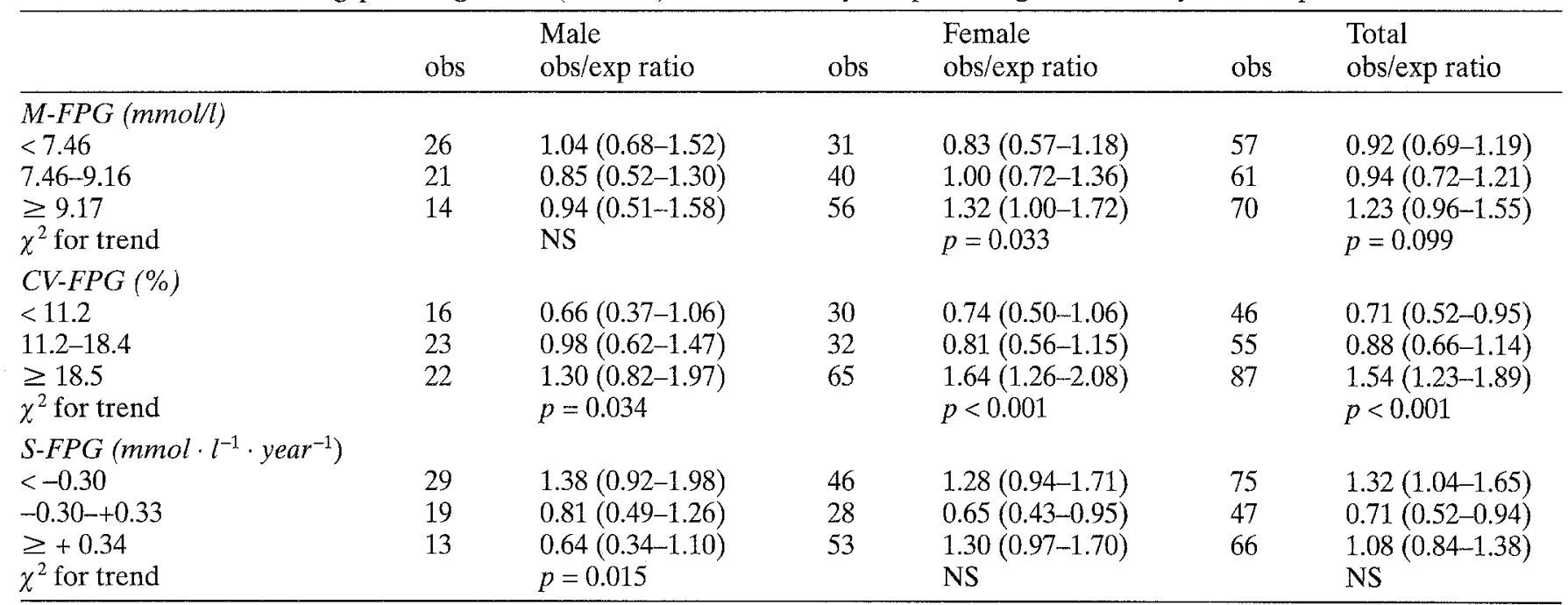

For each level of M-FPG, CV-FPG, and S-FPG the number of observed deaths (obs) and the observed/expected (obs/exp) ratio with the $95 \%$ confidence intervals are reported. Significance was assessed through a chi-square for trend. NS, Not significant

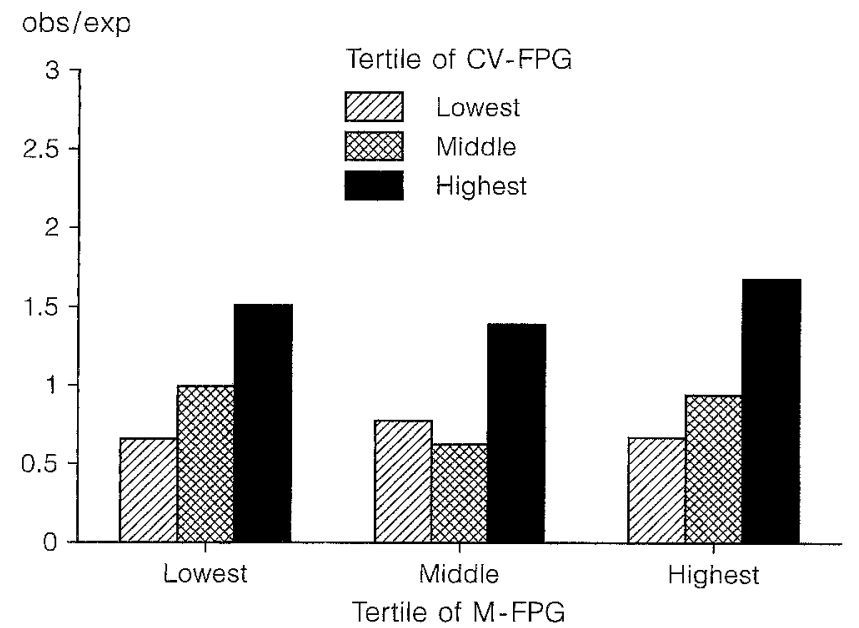

Fig.1. Observed/expected death ratio (obs/exp) in subjects grouped in tertiles according to mean fasting plasma glucose (M-FPG) as well as coefficient of variation of fasting plasma glucose (CV-FPG) during the 3 years (1 January 1984 through 31 December 1986) preceding a 5-year (1 January 1987 to 31 December 1991) mortality follow-up. No significant differences in obs/exp were found in tertiles of M-FPG, while a significant increase was found across tertiles of CV-FPG

\section{Results}

By the end of the 5-year follow-up 188 patients (61 men, 127 women) had died. As shown in Table 2, patients who died were slightly but significantly older, and had higher M-FPG and CV-FPG, but not SFPG, during the 3-year period of retrospective analysis of fasting plasma glucose. Even though the overall therapy pattern did not reach statistical significance, patients who died were treated with insulin (alone or with oral hypoglycaemic agents) more frequently than survivors. For this reason, insulin treatment was included in the multivariate survival analysis (see below).

As shown in Table 3, patients treated with insulin, alone or in combination with oral hypoglycaemic agents, displayed significantly greater M-FPG and CV-FPG during the 3-year period preceding the mortality follow-up as compared with patients treated with diet or oral agents. S-FPG was not significantly affected by the treatment.

Table 4 reports the observed/expected ratio during the 5-year mortality follow-up (1 January 1987-31 December 1991), as a function of M-FPG, CV-FPG and S-FPG during the period of retrospective evaluation of fasting plasma glucose. A significant increase in observed/expected ratio was observed in diabetic women of the top tertile of M-FPG, but not in men or in the whole cohort, where the trend for an increased mortality with the increase of M-FPG did not reach statistical significance $(p=0.099)$.

A significant increase in observed/expected ratio was found as a function of CV-FPG in men, in women and in the whole cohort. In particular, patients of the top tertile of CV-FPG (i.e., those with fasting plasma glucose instability) experienced significant excess mortality as compared to the non-diabetic population. Interestingly, patients of the lowest CVFPG tertile showed a significantly decreased mortality as compared with the non-diabetic population (Table 4).

S-FPG affected mortality risk in a non-linear fashion. Indeed, mortality risk was significantly increased in the lowest tertile (i.e., subjects with a trend toward lower glucose levels over time), and was significantly reduced in the middle tertile (i.e. subjects with a glu- 
Table 5. Relative risk (with $95 \%$ confidence intervals in parentheses) of death from all causes in the elderly diabetic patients, after adjustment for age and sex, and after adjustment for all other variables

\begin{tabular}{|c|c|c|c|}
\hline \multirow[t]{2}{*}{ Variables } & \multicolumn{2}{|l|}{ Relative risk } & \multirow[t]{2}{*}{$p^{\text {a value }}$} \\
\hline & $\begin{array}{l}\text { Adjusted for age and sex, } \\
\text { if not in the model }\end{array}$ & $\begin{array}{l}\text { Adjusted for all } \\
\text { other variables }\end{array}$ & \\
\hline $\begin{array}{l}\text { Sex (women vs men) } \\
\text { Age }(\mathrm{SD}=2.6 \text { years) } \\
\text { Diabetes duration }(\mathrm{SD}=7.0 \text { years) } \\
\text { Insulin treatment (yes vs no) }\end{array}$ & $\begin{array}{l}0.76(0.56-1.04) \\
1.16(1.01-1.33) \\
0.99(0.86-1.15) \\
1.97(1.22-3.17)\end{array}$ & $\begin{array}{l}0.71(0.52-0.97) \\
1.16(1.00-1.35) \\
0.95(0.82-1.10) \\
1.61(0.96-2.72)\end{array}$ & $\begin{array}{l}=0.033 \\
=0.049 \\
=0.460 \\
=0.086\end{array}$ \\
\hline $\begin{array}{r}\text { M-FPG (II vs I tertile) } \\
\text { (III vs I tertile) }\end{array}$ & $\begin{array}{l}1.06(0.74-1.53) \\
1.31(0.92-1.87)\end{array}$ & $\begin{array}{l}0.91(0.63-1.32) \\
0.94(0.64-1.39)\end{array}$ & $=0.880$ \\
\hline $\begin{array}{r}\text { CV-FPG (II vs I tertile) } \\
\text { (III vs I tertile) }\end{array}$ & $\begin{array}{l}1.22(0.83-1.81) \\
2.18(1.52-3.12)\end{array}$ & $\begin{array}{l}1.16(0.77-1.74) \\
1.91(1.28-2.85)\end{array}$ & $=0.002$ \\
\hline $\begin{array}{l}\text { S-FPG (II vs I tertile) } \\
\text { (III vs I tertile) }\end{array}$ & $\begin{array}{l}0.58(0.40-0.83) \\
0.85(0.61-1.19)\end{array}$ & $\begin{array}{l}0.75(0.51-1.13) \\
0.91(0.65-1.28)\end{array}$ & $=0.380$ \\
\hline
\end{tabular}

${ }^{a}$ Significance of each variable in the Poisson regression model after adjusting for all other variables

M-FPG, CV-FPG and S-FPG are, respectively, the mean, coefficient of variation and slope of fasting plasma glucose. Rela-

tive risks and significance of differences were derived from Poisson regression analyses. Calculation of the relative risk for continuous variables was based on an increase in the values of 1 SD
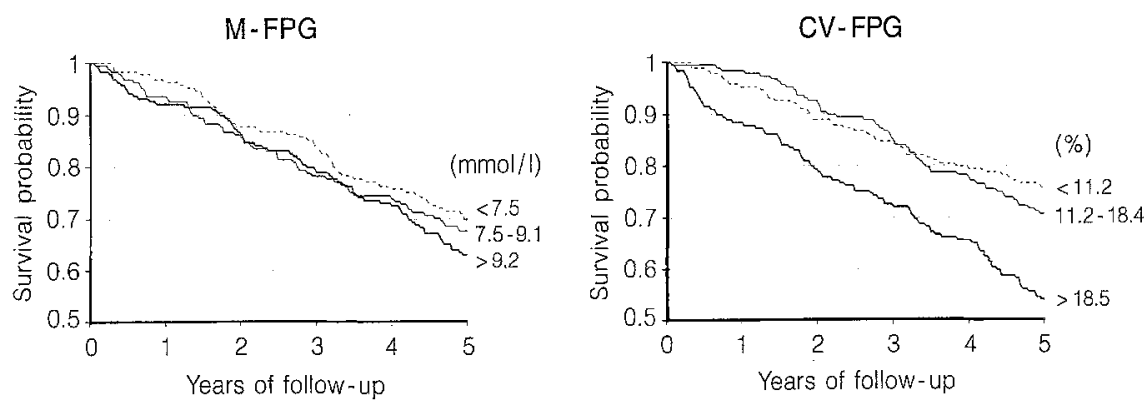

Fig. 2. Kaplan-Meier estimates of survival probability in 566 elderly NIDDM patients from Verona, 1 January 1987 through 31 December 1991. Patients were grouped in tertiles according to mean fasting glucose (M-FPG), coefficient of variation of fasting

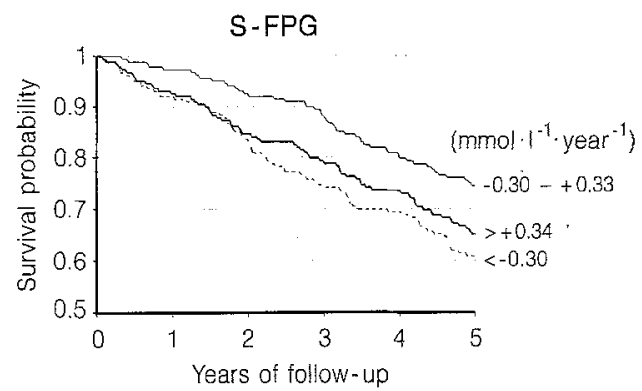
plasma glucose (CV-FPG) and slope of fasting plasma glucose over time (S-FPG) during the 3 years (1 January 1984 through 31 December 1986) preceding a 5-year (1 January 1987 to 31 December 1991) mortality follow-up. The log-rank test revealed significant differences in survival between tertiles of CV-FPG $(p<0.001)$ and S-FPG $(p<0.01)$

cose level not substantially changing over time) (Table 4).

Figure 1 depicts the mortality risk as a function of both M-FPG and CV-FPG. It is evident that the increase in M-FPG, irrespective of the category of CVFPG, did not result in an increase in mortality, while the increase in CV-FPG did, in all categories of $\mathrm{M}$ FPG.

The survival analysis by the Kaplan-Meier method was carried out as a function of M-FPG, CV-FPG and S-FPG during the 3 years preceding the mortality follow-up (Fig. 2). This analysis confirmed the results shown in Table 4 and Fig.1, indicating that in the overall cohort M-FPG had a marginal effect on mortality, while CV-FPG and, to a minor extent, S-FPG were significant predictors of mortality. In particular, subjects with increased mortality were those in the top tertile of CV-FPG and those in tertiles I and III of S-FPG (i.e., tertiles representing subjects with a trend toward higher or lower values of plasma glucose over time).

Poisson regression model (Table 5) showed that gender, age, and CV-FPG during the 3 years preceding the mortality follow-up were significant predictors of mortality. Insulin treatment and S-FPG were significant predictors of mortality only when gender and age, but not other variables, were included in the model.

A similar analysis, carried out in a subset of 399 subjects for an extended period (11 years) of retrospective evaluation of fasting plasma glucose, yielded superimposable results (data not shown). 


\section{Discussion}

In the present study all-cause mortality was assessed prospectively over a period of 5 years (1987-1991) in a cohort of 566 elderly patients with NIDDM. The purpose of the study was to evaluate the relationships between mortality rates and long-term metabolic control, as assessed by the retrospective analysis of all fasting plasma glucose values available in the clinical records of each individual subject and measured in the years preceding the mortality follow-up.

Mean fasting plasma glucose (M-FPG), coefficient of variation of fasting plasma glucose (CV-FPG) and the slope of fasting plasma glucose levels over time (S-FPG) in the 3 years (1984-1986) preceding the mortality follow-up were calculated in each individual subject.

Analysis of mortality, based on sex- and age-adjusted observed/expected ratios, and univariate survival analysis carried out by calculating Kaplan-Meier survival curves, revealed a significant excess mortality in women in the top tertile of M-FPG, and an increase of mortality with increasing CV-FPG. Also, subjects of tertiles I and III of S-FPG, i.e. those with a trend toward lower or higher plasma glucose levels over time, showed a significant increase in mortality. Multivariate survival analysis by Poisson regression model showed that only CV-FPG exerts an independent effect on all-cause mortality.

Interestingly, while CV-FPG was an independent predictor of mortality, the mean fasting plasma glucose and the slope of fasting plasma glucose over time were not. This might suggest that the instability of metabolic control, more than the severity of hyperglycaemia or the progression toward higher or lower fasting plasma glucose levels over time, has an effect on survival in elderly diabetic subjects. However, it should be noticed that many subjects under study of the top tertile of M-FPG had a metabolic control still in the "fair" range and this might mask the effect of hyperglycaemia on mortality. Uusitupa et al. [17] have recently observed a significant effect of hyperglycaemia on mortality. However, these authors did not evaluate glucose variability and examined younger subjects who had glucose levels consistently higher $($ mean $=11.1 \mathrm{mmol} / \mathrm{l})$ than subjects of our study (mean $=8.5 \mathrm{mmol} / \mathrm{l}$ ). In this respect, we were able to find an effect of M-FPG on mortality in subjects with $\mathrm{M}-\mathrm{FPG}>11.1 \mathrm{mmol} / \mathrm{l}(200 \mathrm{mg} / \mathrm{dl})$ but only when the multivariate analysis did not include CVFPG.

While several studies have reported that NIDDM is associated with an excess mortality for all causes $[6,9,10,17-19)$, only few data are available about the relationship existing between mortality and degree of metabolic control. As previously mentioned, a significant effect of the severity of hyperglycaemia on mortality in NIDDM has been reported by Uusi- tupa et al. [17]. For 10 years these authors followedup 133 newly-diagnosed NIDDM patients with a mean age of $\sim 55$ years, and found that fasting plasma glucose values at baseline, and fasting plasma glucose and $\mathrm{HbA}_{1 \mathrm{c}}$ values at 5 years were higher in diabetic subjects who died during the period of observation than in those who survived. In our study the experimental design was different from that of the study by Uusitupa et al. [17]. Indeed, while these authors considered a single value of fasting plasma glucose at baseline and at 5-year follow-up, and a single determination of $\mathrm{HbA}_{\mathrm{Ic}}$ at 5-year follow-up, we retrospectively analysed several fasting plasma glucose determinations over 3 years and evaluated the mortality in the subsequent 5 years. It is conceivable that our methodological approach gives more reliable information about long-term glucose control. In addition, our study provides original information on the relationship between mortality and plasma glucose variability. To our knowledge, there are currently no other data available on the effect of metabolic control on mortality in NIDDM subjects. Moreover, we are not aware of any study reporting the association between mortality and plasma glucose variability (i.e., glucose stability) in diabetes. Most probably two ongoing large national surveys (United Kingdom Prospective Diabetes Study and Veterans Administration Collaborative Diabetes Study) will provide exhaustive data on this issue, but these studies are still in progress $[20,21]$.

The relationship between mortality and the severity of hyperglycaemia is generally explained by the notion that hyperglycaemia is a major determinant of chronic diabetic complications [22-25] and that hyperglycaemia worsens the outcome of several diseases $[26,27]$. More difficult to interpret is the relationship between mortality and glucose variability. This relationship could have several different interpretations. However, a number of potential biases need to be preliminaril considered. First, variability in fasting plasma glucose was not a marker of a progressive deterioration in metabolic control, as the slope of fasting plasma glucose levels over time (S-FPG) was poorly correlated to CV-FPG $\left(R^{2}=0.03\right)$ and was not independently associated with excess mortality in the multivariate analysis. Secondly, glucose variability was not an epiphenomenon of poor glycaemic control, since there was a weak correlation between M-FPG and CV-FPG $\left(R^{2}=0.06\right)$.

The mechanisms relating glucose variability with mortality are unknown, but a set of hypotheses could be formulated. First, glucose variability could indicate erratic compliance to diabetes treatment or could be the expression of recurrent relapses of coexisting illnesses, which might be eventually responsible for death. Glucose variability could also be a condition predisposing to hypoglycaemia, which is a wellknown risk factor for severe adverse outcomes, such 
as trauma, stroke, myocardial infarction and arrhythmia [28]. Finally, glucose variability may precipitate the events leading to mortality through diabetes-related or non-diabetes-related mechanisms.

One might be concerned about the fact that we have used repeated fasting plasma glucose and not repeated glycated haemoglobin determinations, which might provide a better estimate of overall metabolic control. Unfortunately, in most subjects of our cohort data on glycated haemoglobin during years 1984-1986 were not available because this parameter in that period was routinely assessed only in insulin-treated or in unstable subjects. However, we believe that a repeated measure of fasting plasma glucose is a sufficiently reliable tool to evaluate the degree of metabolic control.

Another concern might be related to the fact that we have not measured fasting plasma glucose during the years of mortality follow-up and, consequently, we cannot exclude that the degree of metabolic control in the months immediately preceding the death might have been substantially different from that of the period of retrospective analysis of fasting plasma glucose. This concern would be especially stringent for subjects who died during the last year of followup. However, in the Kaplan-Meier analysis we observed that the effect of high coefficient of variation on mortality was appreciable to the same extent in the initial and the final part of the follow-up. Moreover, when survival analysis with the Poisson regression model was restricted to the first year of followup (1987) or to the last year of follow-up (1991), CVFPG remained an independent statistical predictor of mortality.

In the present study the duration of disease was rather long, and one could raise the question on whether a shorter duration of diabetes might modify the results. Indeed, it might be hypothesized that the degree of metabolic control (e.g., instability) may not be related to mortality risk when diabetes of elderly subjects has a short duration. However, in the multivariate model CV-FPG proved to be a significant predictor of mortality independently of the duration of diabetes.

The impact of glucose control on mortality might not be independent of other cardiovascular risk factors. A complete profile of cardiovascular risk factors was available only in about $50 \%$ of subjects under study. When data on these cardiovascular risk factors (blood pressure, plasma lipids, body mass index) were included in the multivariate analysis, CV-FPG remained an independent significant predictor of mortality. However, it is important to stress that in the present study we examined all-cause mortality and not cardiovascular mortality.

Over the last few years several studies have raised the question whether endogenous hyperinsulinaemia [29] and/or insulin treatment [30,31] are associated with cardiovascular disease and, eventually, mortality. In our study, we observed that subjects treated with insulin had sex- and age-adjusted relative risk of death higher than those not treated with insulin. However, this risk, although still remarkable (1.61), was no longer statistically significant when the regression model included M-FPG, CV-FPG and S-FPG. The loss of significance when all variables were included in the model might be merely a consequence of the small number of insulin-treated subjects in the cohort. Alternatively, insulin treatment may be associated with excess mortality because it was given to subjects with the worse metabolic control, rather than being a direct effect of insulin. In other words, insulin treatment given to control glucose levels would track hyperglycaemia and/or glucose variability.

The present study suggests that in elderly NIDDM mortality, i. e. an unquestionable end-point, is related to glucose variability more than to the degree of metabolic control. The latter point seems to be of particular interest, since tight metabolic control is now recommended to prevent chronic complications in insulin-dependent diabetes [22], and at present there is considerable debate on how the DCCT data can be extended to NIDDM $[32,33]$. Our results suggest that, at least in elderly NIDDM subjects, the target of therapy could be more conservative than in younger insulin-dependent diabetic patients. Indeed, it seems that fasting plasma glucose levels ranging from $7-10 \mathrm{mmol} / 1(140-180 \mathrm{mg} / \mathrm{dl})$ are acceptable in terms of survival probability, provided that glucose variability is kept as low as possible. In conclusion, our data suggest that glucose stability needs to be considered along with the level of metabolic control when treating elderly NIDDM patients.

Acknowledgements. This study was supported by grants No. 86.01873.56, 87.00374.56, 91.00400.PF40, 92.00337.PF40, 93.00421.PF40 from the Italian National Research Council (INV 953540) and by grants from the Italian Ministry of University and Scientific and Technological Research.

The authors are grateful to Dr. C. Zamboni, G. Manfrin and G. Nota of the Department of Statistics and Informatica of the Verona SHU.

\section{References}

1. Harris MI, Hadden WC, Knowler WC, Bennet PH (1987) Prevalence of diabetes and impaired glucose tolerance and plasma glucose levels in U.S. population aged 20-74 yr. Diabetes 36: 523-534

2. Neil HAW, Gatling W, Mather HM et al. (1987) The Oxford Community Diabetes Study: evidence for an increase in the prevalence of known diabetes in Great Britain. Diabet Med 4: 539-543

3. Damsgaard EM, Faber OK, Froland A, Green A, Hauge M, Holm NV, Iversen S (1987) Prevalence of fasting hyperglycaemia and known non-insulin-dependent diabetes mellitus classified by plasma C-peptide. Diabetes Care 10: 26-32 
4. Croxon SCM, Burden AC, Bodington M, Botha JL (1991) The prevalence of diabetes in elderly people. Diabet Med 8: $28-31$

5. Bruno G, Bargero G, Vuolo A, Pisu E, Pagano G (1992) A population-based prevalence survey of known diabetes mellitus in Northern Italy based upon multiple independent sources of ascertainment. Diabetologia 35: 851-856

6. Muggeo M, Verlato G, Bonors E et al. (1995) The Verona Diabetes Study: a population-based survey on known diabetes mellitus prevalence and 5-year all-cause mortality. Diabetologia 38: 318-325

7. Cohen DL, Neil H, Thorogood M, Mann JI (1991) A population-based study of the incidence of complications associated with type 2 diabetes in the elderly. Diabet Med 8: 928 933

8. Halter JB (1994) Geriatric patients. In: Lebovitz HE (ed) Therapy for diabetes mellitus and related disorders, 2nd edn. American Diabetes Association, Alexandria, VA, USA, pp 164-169

9. Kleinmann JC, Donahue RP, Harris MI, Finucane FF, Madans JH, Brock DB (1988) Mortality among diabetics in a national sample. Am J Epidemiol 128: 389-401

10. Stengard JH, Tuomilehto J, Pekkanen J et al. (1992) Diabetes mellitus, impaired glucose tolerance and mortality among elderly men: the Finnish cohorts of the Seven Countries Study. Diabetologia 35: 760-765

11. Entmacher PS, Sinnock P, Bostic E, Harris MI (1985) Economic impact of diabetes. In: National Diabetes Data Group (ed) Diabetes in America; NIH Publication No. 85-1468, pp XXXII.1-13

12. National Diabetes Data Group (1979) Classification and diagnosis of diabetes mellitus and other categories of glucose intolerance. Diabetes 28: 1039-1057

13. Coleman M., Douglas A, Hermon C, Peto J (1986) Cohort study analysis with a Fortran computer program. Int J Epidemiol 15: 134-137

14. Kaplan EL, Meier P (1958) Nonparametric estimation from incomplete observations. J Am Stat Assoc 53: 457-481

15. Clayton D, Hills M (1993) Statistical models in epidemiology. Oxford Science Publication, Oxford (UK)

16. Snedecor GW, Cochran WG (1967) Statistical methods. Ames Iowa, Iowa State University Press

17. Uusitupa MIJ, Niskanen LK, Siitonen O, Voutilainen E, Pyorala K (1993) Ten-year cardiovascular mortality in relation to risk factors and abnormalities in lipoprotein composition in type 2 (non-insulin-dependent) diabetic and nondiabetic subjects. Diabetologia 36: 1175-1184

18. Fuller JH, Elford J, Goldblatt P, Adelstein AM (1983) Diabetes mortality: New light on an underestimated public health problem. Diabetologia 24: 336-341
19. Fuller JH (1994) Mortality from diabetes. In: Williams R, Papoz L, Fuller JH (eds) Diabetes in Europe. Libbey, London, pp 108-116

20. UK Prospective Diabetes Study Group (1991) UK prospective diabetes study (UKPDS). VIII. Study design, progress and performance. Diabetologia 34: 877-890

21. Abraira C, Emanuele N, Colwell J, Henderson W, Comstock J, Lvin S, Nuttal F, Sawin C, VACS Group (1992) Glycaemic control and complications in type II diabetes: design of the feasibility trial. Diabetes Care 15: 1560-1571

22. Diabetes Control and Complications Trial Research Group (1993) The effect of intensive treatment of diabetes on the development and progression of long-term complications in insulin-dependent diabetes mellitus. N Engl J Med 329: 977-986

23. Reichard P, Nilsson BY, Rosenqvist U (1993) The effect of long-term intensified insulin treatment on the development of microvascular complications of diabetes mellitus. N Engl J Med 329: 304-309

24. Raskin P, Rosenstock J (1986) Blood glucose control and diabetic complications. Ann Intern Med 105: 254-263

25. Liu QZ, Pettitt DJ, Hanson RL, Charles MA, Klein R, Bennett PH, Knowler WC (1993) Glycated haemoglobin, plasma glucose and diabetic retinopathy: cross-sectional and prospective analyses. Diabetologia 36: 428-432

26. Wilson RM (1991) Infections and diabetes mellitus. In: Pickup J, Williams G (eds) Textbook of diabetes. Blackwell Scientific Publications, Oxford, pp 813-819

27. Gill GV (1991) Surgery and diabetes mellitus. In: Pickup J, Williams G (eds) Textbook of diabetes. Blackwell Scientific Publications, Oxford, pp 820-826

28. Frier BM (1993) Hypoglycemia in the diabetic adult. Clin Endocrinol Metab 7: 757-777

29. Jarrett RJ (1988) Is insulin atherogenic? Diabetologia 31: 71-75

30. Janka HU, Ziegler AG, Standl E, Mehnert H (1987) Daily insulin dose as a predictor of macrovascular disease in insulin treated non-insulin-dependent diabetics. Diabete Metabol 13: 359-364

31. Uusitupa MIJ, Niskanen LK, Siitonen O, Voutilainen E, Pyorala K (1990) 5-year incidence of atherosclerotic vascular disease in relation to general risk factors, insulin levels, and abnormalities in lipoprotein composition in non-insulin-dependent diabetes and non-diabetic subjects. Circulation 82: 27-36

32. Eastman RC, Siebert CS, Harris M, Gorden P (1993) Clinical Review 51. Implications of the Diabetes Control and Complications Trial. J Clin Endocrinol Metab 77:1105-1107

33. Lebovitz HE (1994) The DCCT and its implications for NIDDM. Clinical Diabetes 12: 3-4 